\title{
Spécificités des milieux urbain et rural : une étude comparative sur les résultats du projet At Home / Chez Soi au Nouveau-Brunswick
}

\author{
Danielle Nolin \\ Université de Moncton \\ Jonathan Jetté \\ Université d'Ottawa
}

\begin{abstract}
RÉSUMÉ
Le site de Moncton se trouve à être la plus petite des 5 villes canadiennes participant au projet de recherche At Home / Chez Soi visant à expérimenter et évaluer le modèle Logement d'abord. Une des conséquences de cette petite taille est la proximité et l'importance de l'environnement rural. Nous avons ajouté un sous-projet rural $(n=22)$ au volet qualitatif pour la région de Moncton $(n=20)$. Nous procédons à une analyse comparative de ces 2 volets de façon à mettre en évidence les opportunités et les contraintes propres à chacun, surtout liés aux transports et à la concentration des services.
\end{abstract}

Mots clés : santé mentale, rural, logement

Danielle Nolin, Professeure en Éducation à l'Université de Moncton; Jonathan Jetté, étudiant diplômé dans le programme de psychologie clinique à l'Université d'Ottawa.

Nous remercions Jayne Barker (2008-11), Cameron Keller (2011-12), et Catharine Hume (2012-présent), directrices et directeur nationales du projet At Home / Chez Soi de la Commission de la santé mentale du Canada, ainsi que Paula Goering, directrice nationale de la recherche pour le projet, l'équipe nationale de la recherche, les cinq équipes de chercheurs des sites, les coordonnateurs de site, et les nombreux fournisseurs de services et de logements, et les personnes ayant vécu des expériences qui ont contribué à ce projet et la recherche. Cette recherche a été rendue possible grâce à la contribution financière de Santé Canada à la Commission de la santé mentale du Canada. La Commission de la santé mentale du Canada a géré le développement et l'implantation de l'étude et a fourni la formation et le soutien technique aux équipes d'intervenants et d'intervenantes et aux équipes de chercheurs impliquées dans le projet. Les opinions exprimées représentent uniquement celles des auteurs.

La correspondance concernant cet article devrait être acheminée à Danielle Nolin, Professeure en Éducation, Université de Moncton, Campus de Shippagan, Pavillon Irène-Léger, 218, boul. J.-D.-Gauthier, Shippagan (Nouveau-Brunswick), E8S 1P6. Tél : (506) 336-3400 poste 8208. Téléc : (506) 336-3477. Courriel : danielle.nolin@umoncton.ca 


\begin{abstract}
The Moncton site is the smallest of 5 Canadian cities that participated in the At Home / Chez Soi research project. The project evaluated the implementation and effectiveness of the Housing First model. A unique feature of Moncton was its proximity to rural communities. Therefore, it was decided to add a rural subproject to the qualitative component of the evaluation. A total of 22 rural participants and 20 participants from Moncton were interviewed. Comparative analyses of these 2 groups were conducted. Results demonstrated the opportunities and constraints of living in each area, mainly related to transportation and access to services.
\end{abstract}

Keywords: mental health, rural, housing

L'approche Logement d'abord (Housing First), popularisée par l'équipe de Pathways (Tsemberis, 1999), a gagné en popularité au cours des dernières années en tant qu'intervention pour venir en aide aux personnes sans-abri avec des problèmes de santé mentale. L'intervention a capté l'attention des politiques canadiennes au point où la Commission de la santé mentale du Canada a financé le projet At Home / Chez Soi, une étude de démonstration pancanadienne de cinq sites. L'intervention à la plus petite de ses villes, Moncton, avait également une composante rurale. Cette article compare les résultats de l'étude de Moncton avec ceux de la sous-étude rurale.

À ce jour de nombreuses publications ont permis de documenter l'efficacité de l'intervention Logement d'abord au plan de la stabilisation du logement (O'Connell, Kasprow et Rosenheck, 2009; Stefancic et Tsemberis, 2007), de la diminution d'hospitalisations (Gulcur, Stefancic, Shinn, Tsemberis et Fischer, 2003; O'Connell et al., 2009) et de l'intégration communautaire (O'Connell et al., 2009; Rosenheck, Kasprow, Frisman et LiuMares, 2003). Une certaine quantité de recherches qualitatives ont également exploré la question sous divers angles, soit sur le plan de l'efficacité de l'intervention pour ce qui est de la réduction de la consommation de substances (Padgett, Stanhope, Henwood et Stefancic, 2011), soit pour explorer l'expérience des participants (hommes et femmes) débutant un programme Logement d'abord (Henwood et al., 2013; Polvere, Macnaughton et Piat, 2013).

À notre connaissance, il n'existe aucune étude ayant testé l'approche Logement d'abord en région rurale. En se basant sur les estimations américaines récentes, le problème de l'itinérance est bien réel en région rurale, puisque 14 personnes sur 10000 y seraient sans-abri, comparativement à 29 sur 10000 pour les régions urbaines (Henry et Sermons, 2010). La prévalence des difficultés de santé mentale semble également être différente en région rurale (Judd et al., 2002). Une recherche sur les services de santé mentale disponibles pour les personnes sans logement en région rurale a démontré le manque de services et principalement de services proactifs adaptés à cette population (Forchuk et al., 2010). Ainsi, la flexibilité du modèle Logement d'abord pourrait s'avérer un atout essentiel pour l'offre de services proactifs en région rurale.

Néanmoins, il n'est pas assuré qu'une intervention qui fonctionne en région urbaine ait les mêmes répercussions en région rurale, puisque la réalité des deux populations est différente. Notamment, l'accès aux services et aux ressources est différent (Forchuk et al., 2010). L'offre de services gagne à tenir compte de 
défis additionnels tels que l'accessibilité aux transports et l'éloignement des services. Les attitudes différentes à l'égard des services sociaux, ainsi que les dynamiques d'exclusions sociales doivent être également prises en considération (Forchuk et al., 2010). De plus, la distance à parcourir par l'équipe de soins ainsi que les difficultés à lier les clients et clientes aux ressources doivent être prises en considération pour les services de suivi en communauté offerts en région rurale (Wane, Owen, Sood, Bradley et Jones, 2007). Finalement, l'équipe de Grigg, Judd, Ryan, et Komiti (2004) a démontré que le bassin de logements abordables en région rurale était différent de celui des régions urbaines en ce qui concerne le choix, la quantité et la qualité.

Peu de publications existent à ce jour sur des interventions en région rurale pour une population sans-abri avec des problèmes de santé mentale (Meyer et Morrissey, 2007). Une étude par Prabhu et Browne (2007) a démontré qu'un modèle de services par équipe multidisciplinaire pouvait être offert en région rurale. Leurs participants ont rapporté des gains dans multiples sphères de leur vie suite à un questionnaire autorapporté mis sur pied pour l'étude. Une étude effectuée par Wane et al. (2007) a également testé l'implantation d'un modèle similaire ayant pris place à la fois dans une région rurale et une région urbaine. Leurs analyses ont démontré une réduction de l'hospitalisation au cours de l'étude. Une revue de la littérature récemment effectuée par Meyer et Morrissey (2007) sur les suivis intensifs multidisciplinaires a permis d'identifier que la majorité des études qui ont utilisé un modèle de traitement en région rurale se sont tournées vers une adaptation d'un modèle de suivi intensif multidisciplinaire.

À ce jour, les études sur les services en région rurale se sont principalement intéressées à des populations avec des problèmes de santé mentale sans historique de difficulté résidentielle. De là vient probablement le fait qu'aucune étude répertoriée n'ait utilisée l'approche Logement d'abord. Finalement, les études répertoriées ne documentent pas l'ensemble des changements ni en les comparant à un groupe les recevant en région urbaine, ni en documentant le processus à travers le temps pour un groupe ne recevant pas d'intervention spécifique. Notre recherche adressera l'ensemble de ces lacunes identifiées dans la littérature. À l'aide d'une méthode qualitative comparative nous répondrons aux questions de recherche suivantes :

1. Quels sont les opportunités et les contraintes propres à chacun des groupes d'intervention (région rurale vs urbaine) ayant bénéficié de l'intervention Logement d'abord?

2. Quels sont les opportunités et les contraintes propres à chacun des groupes d'intervention (région rurale vs urbaine) ayant reçu le traitement habituel?

\section{MÉTHODOLOGIE}

\section{Mise en contexte}

Avec une population d'environ 138000 personnes pour la région métropolitaine de Moncton incluant Dieppe et Riverview (New Brunswick Department of Post-Secondary Education, Training and Labour, 2012), le site de Moncton se trouvait à être la plus petite ville participant au projet de recherche At Home / Chez Soi, les autres villes étant Montréal, Toronto, Winnipeg et Vancouver. Une des conséquences de cette petite taille est la proximité et l'importance de l'environnement rural. Nous avons décidé de ce fait d'ajouter un sous-projet rural au volet qualitatif pour la région de Moncton du projet At Home / Chez Soi. 
Le volet qualitatif de Moncton et le sous-projet rural comptaient un nombre égal de participants, partagés entre populations anglophones et francophones. Le volet qualitatif a étudié la population du Grand Moncton, c'est-à-dire la ville de Moncton elle-même ainsi que deux villes de banlieue, soit Dieppe et Riverview, tandis que le volet rural s'est intéressé aux diverses communautés situées dans l'environnement immédiat de ces trois centres urbains dans les comtés de Kent et Westmorland. Il s'agit d'un peuplement linéaire par sa répartition géographique le long de la côte et rural par sa composition en petits villages. Une forte proportion des petites localités de la côte sont composées des communautés acadiennes. Le Sud-Est comprend aussi une importante communauté mi'kmaq.

\section{Le recrutement}

Milieu urbain. Le recrutement que nous avons effectué pour le milieu urbain s'est fait à partir de la base de données fournie par la coordonnatrice de terrain de l'équipe quantitative du projet Chez Soi. Les références provenaient des organismes communautaires, des milieux hospitaliers, des services en santé mentale déjà existants, d'autres organismes œuvrant dans le domaine de la santé et des participants eux-mêmes. L'équipe a rencontré ces personnes pour s'assurer qu'elles rencontrent les critères de sélection, soit : être âgé d'au moins 18 ans, n'avoir aucune personne sous sa responsabilité, avoir des problèmes de santé mentale, être itinérant ou dans une situation de logement précaire (c.à-d., résidant dans une maison de chambres et avoir vécu deux épisodes d'itinérance d'au moins 7 jours consécutifs ou un épisode d'une durée d'un mois durant la dernière année). Notons que le couch surfing, c'est-à-dire une situation de logement précaire chez des parents, ami(e)s ou connaissances, est une situation également retenue aux fins de la recherche. Un questionnaire informatisé a d'abord été administré par un membre de l'équipe de recherche quantitative afin d'obtenir ces informations et de pouvoir ainsi informer le participant quant à son admissibilité au projet. L'assignation subséquente au groupe expérimental ou au groupe de comparaison a été réalisée sur une base aléatoire. Chacun des deux groupes situés à Moncton, expérimental et de comparaison, devait comprendre 100 personnes. En date du 31 mars 2013, le groupe expérimental et le groupe de comparaison comptaient respectivement 98 et 91 personnes suite à des décès et à des désistements.

L'élément le plus important pour le recrutement des personnes auprès desquelles seraient menées les entrevues était de s'assurer de la diversité des participants. À ce titre, trois facteurs ont été considérés de façon prioritaire. Le premier concernait l'âge des participants. Nous voulions obtenir le plus large éventail possible. Nous avons donc recruté des personnes âgées de 19 ans à 62 ans. Le deuxième critère concernait la langue. Nous avons donc recruté 8 participants francophones et 12 participants anglophones. Enfin, le troisième facteur concernait le sexe. Nous avons choisi 10 hommes et 10 femmes. Nous nous sommes finalement retrouvées avec un total de 10 participants dans le groupe expérimental, soit 5 femmes et 5 hommes, et un total de 10 pour le groupe de comparaison, soit 5 femmes et 5 hommes.

Les données démographiques sur les individus qui constituaient notre échantillon au moment du recrutement sont semblables également aux autres sites du projet Chez Soi. Au moment du recrutement de l'échantillon, quatre participants vivaient en colocation dans un contexte où la violence et la consommation faisaient partie du quotidien. Cinq personnes vivaient dans des chambres avec salle de bain commune, deux vivaient chez de la famille, huit habitaient dans la rue ou faisaient du couch surfing. Enfin, une personne vivait en foyer de soins. Une minorité de ces personnes, autant dans le groupe expérimental que dans le groupe de 
comparaison, sont acadiennes. Les participants proviennent de différents milieux et se sont retrouvés dans la région de Moncton par hasard ou pour le travail dans le passé. Toutefois, les participants francophones sont bilingues tandis que les anglophones ne parlent que leur langue maternelle.

Lors des entrevues du $18^{\mathrm{e}}$ mois, il restait neuf participants dans le groupe expérimental et huit, soit quatre femmes et quatre hommes, dans le groupe de comparaison. Les trois participants manquants avaient alors quitté le projet. Du groupe expérimental, cinq personnes sur neuf ont fait l'entretien en français. Du groupe de comparaison, deux des hommes sont francophones tandis que les six autres participants ont préféré faire l'entretien en anglais.

Milieu rural. Le recrutement des participants pour l'étude en milieu rural s'est effectué selon des méthodes et critères analogues. L'identification de participants potentiels vivant avec des problèmes en santé mentale a été faite par l'équipe du Centre de santé mentale communautaire de Richibucto, des personnes bénévoles de Cap-Pelé, des travailleuses sociales du Centre de ressources et de crise familiale Beauséjour de Shédiac et des psychiatres de Moncton. Les participants potentiels devaient avoir reçu un diagnostic de trouble mental. Les troubles recherchés étaient ceux de l'axe I du DSM-IV-TR (Société américaine de psychiatrie, 2002). Différemment des participants à Moncton, ceux du milieu rural résidaient dans des foyers de soins ou avec leurs familles, ou étaient itinérants ou dans une situation de logement précaire (c.à-d., résidant dans une maison de chambres et avoir vécu un épisode d'itinérance récente).

Pour les besoins de l'étude quantitative rurale, le groupe expérimental était composé de 24 participants et celui de comparaison comprenait 19 participants, ce qui permit d'obtenir un échantillon diversifié au niveau du statut résidentiel, représentatif de la réalité de l'itinérance en milieu rural : on a récruté 10 participants issus du milieu familial, 5 qui vivaient en maisons de chambres, 5 en foyers de soins, 3 sans-abri et un dont le milieu résidentiel est non spécifié. Les 24 participants du groupe expérimental en milieu rural, dont 7 femmes, habitent entre les localités de Saint-Louis-de-Kent et de Cap-Pelé inclusivement. Le territoire couvert, en incluant les localités situées à l'intérieur des terres, est d'environ 140 kilomètres.

Pour le groupe de comparaison, le personnel du Centre de santé mentale communautaire de Richibucto a fait le recrutement en pairage à partir de la liste de participants du groupe expérimental en tenant compte du sexe, de l'âge, de la situation de logement et du type de diagnostic (psychotique ou non). Les troubles de santé mentale recherchés étaient les mêmes que pour le groupe expérimental. En juin 2011, 19 participants formaient le groupe de comparaison, soit 8 qui demeuraient avec leur famille, 9 vivant en foyers de soins, un vivant seul sur une réserve de première nation et un dont le milieu résidentiel est non spécifié.

Pour l'étude qualitative, 11 participants de chacun des groupes, dont 4 femmes et 7 hommes pour le groupe expérimental et 5 femmes et 6 hommes pour le groupe de comparaison, ont été recrutés pour les entrevues du récit. À l'entrevue du $18^{\mathrm{e}}$ mois, il a été possible de réaliser une entrevue avec les 11 participants du groupe expérimental et 8 participants du groupe de comparaison, dont 3 femmes. La sélection des participants a été réalisée par un membre de l'équipe de recherche au printemps 2010.

Processus de recrutement. Le repérage des personnes pour le milieu urbain a eu lieu une fois par mois à raison de quatre personnes sélectionnées à la fois. La stratégie consistait à rencontrer à chaque mois deux personnes du groupe expérimental et deux personnes du groupe de comparaison. Nous avons procédé ainsi jusqu'à l'obtention des 20 participants. Le recrutement a débuté en avril 2009 et s'est terminé en décembre 
2010. Il y a eu une période d'arrêt du recrutement durant la période estivale à la demande de l'équipe de suivi intensif. Pour le milieu rural, le repérage a été effectué un à un, d'abord pour le groupe expérimental et ensuite pour le groupe de comparaison, au fur et à mesure que les participants étaient identifiés par le centre de Richibucto.

Une première collecte de données sous la forme d'une entrevue de récit de vie a été réalisée auprès de chaque participant au fur et à mesure qu'avançait le recrutement. Une seconde entrevue a été menée environ 18 mois plus tard dans le but de mettre en évidence les facteurs de changement et de maintien dans la situation des participants. Les résultats présentés ici proviennent des données de cette seconde série d'entrevues. Les rencontres entre les chercheurs et les participants du groupe expérimental et du groupe de comparaison ont eu lieu dans des cafés ou au bureau de l'équipe de suivi intensif. Les entretiens du $18^{\mathrm{e}}$ mois se sont terminés en décembre 2012.

\section{Conditions de Logement d'abord (groupe expérimental)}

Les conditions du groupe expérimental de notre recherche pour Moncton sont les mêmes que les autres villes du projet Chez Soi (Macnaughton, Goering et Nelson, 2012) et ont obtenu un haut niveau de fidélité au modèle Pathways lors de l'évaluation du site (Macnaughton et al., 2013). Les participants consacrent au plus 30\% de leur revenu pour un logement régulier et privé. Ils ont le choix de l'appartement et du quartier dans lequel ils veulent habiter dans la ville de Moncton. Deux exigences sont reliées à faire partie du groupe expérimental. La première est que le logement doit être payé directement au propriétaire et la deuxième est d'accepter la visite chaque semaine d'un membre de l'équipe de traitement. L'équipe comprend un ratio d'un intervenant ou intervenante pour 10 participants. Elle est composée d'un groupe d'intervention multidisciplinaire spécialisé comprenant travailleurs sociaux et travailleuses sociales, conseillers et conseillères en emploi, spécialistes des dépendances, psychiatres, infirmières, personnes avec expérience vécue, spécialistes de la famille et adjointes et adjoints administratifs, et est dirigée par un chef d'équipe. Ce modèle se trouve à la croisé des services et de la réhabilitation (Marx, Test et Stein, 1973).

\section{Particularités des conditions des soins usuels (groupe de comparaison)}

Les entrevues initiales (Prévost et Nolin, 2011) nous ont appris qu'une des particularités de Moncton est la ruralité francophone qui l'entoure. Toutefois, l'itinérance à Moncton se vit surtout en anglais. Bien que Moncton soit considérée comme une ville bilingue, les organismes communautaires de première ligne utilisent surtout l'anglais pour offrir les services. Nous retrouvons donc des francophones qui n'ont pas les services communautaires dans leur langue maternelle.

\section{Processus de codage et d'analyse}

Le même guide d'entrevue a servi pour les deux volets en respectant sensiblement le même échéancier pour le suivi des entrevues. La différence majeure se situe au niveau de l'analyse des données recueillies. L'analyse des données du volet qualitatif du Grand Moncton a été réalisée selon le principe de l'analyse thématique (Blanchet et Gotman, 1992; Paillé et Mucchielli, 2003), tandis que l'analyse des données du volet rural a été réalisée selon l'approche ethnographique (Fetterman, 1998). Étant donné que l'approche 
ethnographique constitue la plus neutre des deux approches, au sens où le chercheur tend à rendre une description libre d'interprétations de sa part, nous avons appliqué la grille thématique issue de l'analyse du volet qualitatif du Grand Moncton. Ce croisement a permis de mettre en évidence les thèmes récurrents entre les deux sites ainsi que les thématiques singulières propres à chaque site. Une grille avait été suggérée par l'équipe nationale de direction du projet, orientant ainsi les grands thèmes du questionnaire, soit la journée typique, la stabilité du logement, les services, les relations, la santé et le bien-être incluant la médication et la consommation, la situation matérielle, les relations, le travail, le rétablissement et les espoirs pour l'avenir. Chaque étude devait aussi dégager les facteurs qui ont aidé ou gêné les changements ainsi que les facteurs ou qualités qui sont liés à des changements positifs, neutres ou négatifs dans les conditions de traitement.

L'analyse de chaque projet a donc procédé selon une logique à la fois thématique et comparative, puisque pour chacun des thèmes ciblés par le volet national, une comparaison était faite entre les groupes en ce qui concernait leur situation après 18 mois. Cela nous a permis de dégager, pour chacun des thèmes proposés, les éléments propres à chaque groupe ainsi que les éléments communs aux deux.

L'analyse comparative entre les données du volet qualitatif du Grand Moncton et celles du volet rural dont il est question dans le présent texte est donc présentée selon une logique analogue : nous présentons tout d'abord, en fonction de chaque thématique, les réalités communes aux deux sites pour les groupe expérimentaux et, par la suite, les spécificités propres à chaque site. Nous utiliserons le même plan pour présenter les deux groupes de comparaison.

Les éléments de découverte sont aussi exemplifiés par des extraits de verbatims. Chacun de ces extraits est coté de manière à identifier le groupe auquel appartient le participant : $\mathrm{U}=$ volet urbain, $\mathrm{R}=$ volet rural, $\mathrm{E}=$ groupe expérimental, $\mathrm{C}=$ groupe de comparaison. Par exemple, UE désigne un participant du volet urbain et du groupe expérimental; RC désigne un participant du volet rural et du groupe de comparaison.

\section{RÉSULTATS}

\section{Groupes expérimentaux}

Le tableau 1 présente une synthèse des résultats par rapport aux groupes expérimentaux dans les deux contextes.

Éléments communs aux groupes expérimentaux des milieux urbain et rural. Un point commun très fort et très central dans ces études est l'importance pour les participants d'une stabilité du logement :

J'ai une place à vivre, ça c'en est une. (RE 2)

Ah oui. Même si faut que j'paie plus, j'aime ousque jsu, j'aime ça, you know what I mean. (UE 62).

La satisfaction à ce niveau est généralisée, même si on retrouve une certaine stigmatisation de la part de certains propriétaires. Dans tous les cas, les participants considèrent le logement comme une base à partir de laquelle ils sont en mesure de construire des habitudes de vie plus saines et un meilleur bien-être, sans toutefois minimiser la précarité de leurs conditions matérielles. On note d'ailleurs une activité physique plus régulière. Mais, surtout, les participants se sont pour la plupart engagés dans une démarche d'autonomisation, celle-ci passant par une meilleure acceptation de la maladie et une meilleure gestion de leur médication. Cette démarche s'accompagne aussi d'une diminution ou d'une gestion de la consommation de drogues 
Tableau 1

Synthèse des deux groupes expérimentaux

\begin{tabular}{|c|c|c|c|}
\hline Thème & Spécifique à Moncton & $\begin{array}{l}\text { Commun à } \\
\text { Moncton et rural }\end{array}$ & Spécifique à rural \\
\hline $\begin{array}{l}\text { Stabilité du } \\
\text { logement }\end{array}$ & $\begin{array}{l}\text { - Appartenance et } \\
\text { responsabilité } \\
\text { - Stigmatisation quartier }\end{array}$ & $\begin{array}{l}\text { - Satisfaction } \\
\text { - Stigmatisation landlord }\end{array}$ & $\begin{array}{l}\text { - Cohabitation / distance } \\
\text { - Désir d'aller en ville } \\
\text { - Expulsions / prostitution / } \\
\text { drogue } \\
\text { - Achat maison mobile }\end{array}$ \\
\hline $\begin{array}{l}\text { Santé et bien- } \\
\text { être }\end{array}$ & & $\begin{array}{l}\text { - Logement comme base } \\
\text { - Logique d'autonomie } \\
\text { - Acceptation maladie } \\
\text { - Gestion médication } \\
\text { - Diminution / gestion } \\
\text { consommation }\end{array}$ & - Conscience de la médication \\
\hline $\begin{array}{l}\text { Situation } \\
\text { matérielle }\end{array}$ & $\begin{array}{l}\text { - Gestion / économie } \\
\text { argent = autonomie / fierté } \\
\text { - Diversité / régularité de } \\
\text { l'équipe de suivi intensif }\end{array}$ & - Précarité & $\begin{array}{l}\text { - Anxiété liée aux anciennes } \\
\text { dettes }\end{array}$ \\
\hline Journée typique & - Espace de survie & - Activité physique & $\begin{array}{l}\text { - Arts et musique } \\
\text { - Ennui } \\
\text { - Travail }\end{array}$ \\
\hline Services & $\begin{array}{l}\text { - Centralisation accessibilité } \\
\text { des services }\end{array}$ & $\begin{array}{l}\text { - L'équipe de suivi intensif } \\
\text { positif } \\
\text { - Trop d'intervenant(e)s } \\
\text { différents }\end{array}$ & - Initiative médecine douce \\
\hline Relations & $\begin{array}{l}\text { - Survie = méfiance } \\
\text { - Enfants / ami(e)s long + }\end{array}$ & $\begin{array}{l}\text { - Relations fortes }+/- \\
\text { - Nouveaux ami(e)s vs } \\
\text { consommation } \\
\text { - Réseau fragile inexistant }\end{array}$ & $\begin{array}{l}\text { - Ouverture de la communauté } \\
\text { (projets d'insertion) } \\
\text { - Amélioration famille } \\
\text { - Réseau social agrandit }\end{array}$ \\
\hline Travail & $\begin{array}{l}\text { - Projet d'insertion long à } \\
\text { démarrer } \\
\text { - Retour aux études }\end{array}$ & $\begin{array}{l}\text { - Symptômes et stress vs } \\
\text { travail et difficile }\end{array}$ & $\begin{array}{l}\text { - Projets d'insertion } \\
\text { - Manque de transport } \\
\text { - Peu d'emploi } \\
\text { - Bénévolat } \\
\text { - Prostitution }\end{array}$ \\
\hline Rétablissement & & $\begin{array}{l}\text { - Diminution de } \\
\text { l'hospitalisation }\end{array}$ & $\begin{array}{l}\text { - Vieillir et maturation }= \\
\text { acceptation }\end{array}$ \\
\hline $\begin{array}{l}\text { Espoirs pour } \\
\text { l'avenir }\end{array}$ & - Capacité de se voir $=$ & - Projets réalistes & \\
\hline
\end{tabular}


ou d'alcool. À ce titre, la variété et la constance des services de l'équipe de suivi intensif sont considérées comme très importantes :

Je feel que j'ai le support de beaucoup de monde. Juste ça je trouve que mentalement ça fait vraiment du bien, comme de voir qu'ils essayent d'aider. (RE 26)

L'affaire est que, dans tous les services que j'ai là, l'affaire est que j'ai ma liberté [ . . . ] Pis y'a pas aucune argent qui peut l'acheter. (UE 42)

Toutefois, des participants de part et d'autre ont éprouvé des difficultés à composer avec ce qu'ils considéraient comme un trop grand nombre d'intervenantes et d'intervenants différents. Les changements d'intervenantes et d'intervenants ne favorisent pas le lien de confiance et les participants trouvent difficile de devoir recommencer à raconter leur histoire à chaque fois.

La question des relations demeure d'ailleurs un aspect problématique central pour l'ensemble des participants. Les relations familiales jouent ou ont joué un rôle très important pour la plupart des participants, que ce soit de façon positive ou négative. Le réseau social demeure fragile, voire inexistant. Certains participants sont toutefois amenés à se faire de nouveaux amis et amies suite à l'arrêt de la consommation. La possibilité de se trouver un travail fait également partie des problèmes régulièrement rapportés. Les symptômes de la maladie mentale, le stress lié à des échecs vécus précédemment ayant parfois été jusqu'à l'épuisement ou à la dépression, de même que les possibilités limitées de travail qui leur laissent peu de choix quant au type de travail, impliquent presque assurément des conditions trop difficiles pour eux.

Finalement, pour la plupart des participants, le rétablissement passe avant tout par la diminution de l'hospitalisation. On remarque aussi la capacité d'élaborer des projets d'avenir réalistes.

Éléments propres au groupe expérimental du milieu urbain. Le fait que les participants soient en mesure de choisir leur logement et leur quartier amène chez eux un sentiment d'appartenance et de responsabilité. Ceci est amplifié par le fait d'avoir quitté un quartier associé à la pauvreté et la violence :

What do I like the most about my place? Just the fact that I'm [not] downtown where freaks can't come and visit me, idiots that got out of jail. (UE 62)

La centralisation de ces services a aussi été évoquée comme un facteur positif, aspect important ne pouvant être reproduit en milieu rural. En effet, à Moncton, non seulement les services étaient regroupés à une seule adresse, mais ce bâtiment était aussi un endroit où les participants pouvaient venir prendre un café ou faire des recherches d'emploi ou d'autres activités en compagnie des intervenants et intervenantes, et ce tout au long des 3 ans qu'a duré le projet de recherche.

Le changement de rythme de vie par rapport à la survie de l'itinérance crée un sentiment de vide chez certains participants. Le fait d'avoir vécu pendant des années en situation extrêmement précaire rend le calme d'une vie en logement difficile à appréhender. Aussi, les participants du groupe expérimental en milieu urbain ont conservé, suite à ces longs épisodes de survie, une méfiance face aux autres et une difficulté à entrer en contact. Toutefois, on remarque chez ces participants que la présence d'enfants ou d'amis ou amies de longue date constitue un facteur très important dans leur volonté et leur capacité de construire ou rétablir des relations positives. 
Comme le projet d'insertion a été très long à démarrer en milieu urbain, nous n'avons pas été témoins de participants ayant vécu une expérience de travail. Par contre, un participant a connu une expérience positive de retour aux études. Notons que, en ce qui concerne les participants du groupe expérimental, leur capacité d'avoir des projets réalistes dont il a été question précédemment semble liée à une capacité à se voir plus clairement, notamment dans leurs limites :

Euh, j'aimerais avoir une famille, mais euh, c'est difficile euh $[\ldots]$ être capable de $[\ldots]$ parce que quand que moi je pense à avoir une famille j'me dis, est-ce que je vais être capable d'me trouver une femme qui va être capable vivre avec ma maladie. (UE 68)

Éléments propres au groupe expérimental des communautés rurales. En milieu rural, le phénomène du logement offre certaines particularités telles que la cohabitation et le problème omniprésent des distances considérables pour ces personnes ayant une autonomie de locomotion très restreinte. L'ennui est très présent et certains participants expriment d'ailleurs le désir de déménager en ville :

Ça me prendrait de quoi pour me tenir occupé, parce que je passe trop de temps à rien faire, pis là ma mind jump sur le négatif, je trouve les jours assez longs. (RE 17)

Par contre, le milieu rural offre des possibilités, comme un participant qui a été en mesure de faire l'achat d'une maison mobile. Toutefois, parmi les deux groupes expérimentaux, c'est seulement en milieu rural qu'on a vu des expulsions de logement, un pour cause de prostitution et l'autre pour cause de consommation de drogue.

Fait intéressant, on a retrouvé chez certains de ces participants une plus grande conscience de leur médication :

Mes médicaments sont pas mal tout le temps en change, mais les derniers 4 mois j'ai trouvé un bon équilibre dans mes médicaments. $60 \mathrm{mg}$ de Prozac, $30 \mathrm{mg}$ de Abilify 300+25, Seroquel pis le reste, non il reste «Rivotril » pour clonazapam, quatre fois par jour $1 \mathrm{mg}$. (RE 4)

On a aussi remarqué une participante qui a eu l'initiative de se tourner vers la médecine douce. La musique et les arts visuels occupent aussi une place importante dans la vie de plusieurs participants. Ces petits milieux semblent toutefois favoriser un endettement à long terme des participants. Certains de ceux-ci vivent de l'anxiété, étant aux prises avec des anciennes dettes qui les empêchent d'émerger financièrement:

J'ai un problème avec mon crédit, mais j'ai été obligé de renoncer à payer ma dette de crédit jusqu'à temps que ma situation de travail s'améliore. (RE 4)

Le projet d'insertion qui accompagnait le projet Chez Soi a favorisé une ouverture de la communauté envers les participants et a amené ceux-ci à occuper un travail ou à faire du bénévolat. Toutefois, ce projet peut difficilement avoir de suite étant donné les difficultés de transport déjà évoquées conjuguées au peu d'emplois disponibles.

On remarque néanmoins l'amélioration des relations familiales chez certains participants ainsi que l'élargissement du réseau social :

Oui, je m'associe plus avec le monde. C'est ça que j'aime about it, le monde, je vois que le monde me trust, pis ça fait du bien. Je feel plus appreciated. (RE 17)

Pour certains participants, le fait de vieillir amène une maturation qui permet une meilleure acceptation de la maladie. 


\section{Groupes de comparaison}

Le tableau 2 présente une synthèse des résultats parvenant des groupes de comparaison.

Éléments communs aux groupes de comparaison des milieux urbain et rural. Des points communs présents dans les groupes de comparaison, on note avant tout la consommation de drogues et d'alcool ainsi qu'une importante présence des symptômes de la maladie mentale.

Pis j'ai rentrée à l'hôpital le mois de mai, j'étais dans une de mes petites crises. Ça pris six ou sept hommes pour me contrôler. Je ne sais pas quoi ils m'ont donné, j'étais tellement dopée que je m'en rappelle même pas du mois (RC 25).

It's hard to do anything like your daily things $[\ldots]$ and it's hard to have relationships with people. Depression. That's really really the problem, makes me want to just lay in bed all day. (UC 3)

\section{Tableau 2}

Synthèse des deux groups de comparaison

\begin{tabular}{|c|c|c|c|}
\hline Thème & Spécifique à Moncton & $\begin{array}{l}\text { Commun à } \\
\text { Moncton et rural }\end{array}$ & Spécifique à rural \\
\hline $\begin{array}{l}\text { Stabilité du } \\
\text { logement }\end{array}$ & - Insécurité et instabilité & & $\begin{array}{l}\text { - Famille d'accueil / Foyer de } \\
\text { soins - sécurité } \\
\text { - Aucun déménagement }\end{array}$ \\
\hline $\begin{array}{l}\text { Santé et bien- } \\
\text { être }\end{array}$ & $\begin{array}{l}\text { - Isolement pour ne pas } \\
\text { consommer } \\
\text { - Survie } \\
\text { - Logement / services } \\
\text { inadéquats }\end{array}$ & $\begin{array}{l}\text { - Consommation présente } \\
\text { - Symptômes présents } \\
\text { - Portes tournantes }\end{array}$ & $\begin{array}{l}\text { - Effets secondaires } \\
\text { médication }\end{array}$ \\
\hline $\begin{array}{l}\text { Situation } \\
\text { matérielle }\end{array}$ & $\begin{array}{l}\text { - Logique dépendance } \\
\text { - Gestion consommation }\end{array}$ & $\begin{array}{l}\text { - Précarité } \\
\text { - Difficulté à gérer }\end{array}$ & $\begin{array}{l}\text { - Emprunts familles ami(e)s } \\
\text { - Chèque famille d'accueil }\end{array}$ \\
\hline Journée typique & $\begin{array}{l}\text { - Espace de survie } \\
\text { - Dépendance soupe }\end{array}$ & $\begin{array}{l}\text { - Stagnation / routine } \\
\text { - Ennui }\end{array}$ & - Travail \\
\hline Services & & $\begin{array}{l}\text { - Difficulté psychiatres } \\
\text { - Manque de services }\end{array}$ & \\
\hline Relations & - Enfants / ami(e)s long + & $\begin{array}{l}\text { - Survie = méfiance } \\
\text { - Relations fortes }+/- \\
\text { - Réseau fragile inexistant } \\
\text { - Isolement consommation }\end{array}$ & $\begin{array}{l}\text { - Pas à sa place dans } \\
\text { communauté }\end{array}$ \\
\hline Travail & $\begin{array}{l}\text { - Gestion maladie } \\
\text { impossible } \\
\text { - Prostitution }\end{array}$ & & $\begin{array}{l}\text { - Projets d'insertion } \\
\text { - Manque de transport }\end{array}$ \\
\hline \multicolumn{4}{|l|}{ Rétablissement } \\
\hline $\begin{array}{l}\text { Espoirs pour } \\
\text { l'avenir }\end{array}$ & & $\begin{array}{l}\text { - Projets irréalistes ou } \\
\text { inexistants }\end{array}$ & $\begin{array}{l}\text { - Projets réalistes (cesser } \\
\text { fumer / gym) }\end{array}$ \\
\hline
\end{tabular}


Ces deux facteurs contribuent à la mécanique des portes tournantes : la mauvaise gestion de la médication entraine la compensation par la consommation qui aggrave les facteurs de risque liés à la santé mentale. Cela résulte en plus d'hospitalisation et de problèmes avec le système de justice (Lalonde, Grunberg et Aubut, 2001). On note d'ailleurs un manque de services qui se traduit par de très longues listes d'attente. Mais même lorsque ces personnes ont accès à un psychiatre, les difficultés à établir un lien de confiance et à accepter le traitement sont très fréquentes.

Tout comme pour les groupes expérimentaux, la situation matérielle de ces personnes est très précaire. Pour les groupes de comparaison, cette situation est en constante détérioration étant donné les difficultés de gestion du peu de ressources dont ils disposent. On se retrouve ainsi avec des journées empreintes de routine, voire de stagnation et d'ennui. D'ailleurs, le fait d'avoir passé de longues années en situation de survie amène une méfiance généralisée et une difficulté à entrer en contact avec les autres. On retrouve, comme pour les groupes expérimentaux, un réseau social allant de fragile à inexistant, mais certains participants des groupes de comparaison en sont venus à s'isoler encore plus comme seul moyen de contrôler leur consommation. Autre point commun avec les groupes expérimentaux, la famille a joué ou joue encore un rôle majeur, que ce soit en bien ou en mal.

En terminant, lorsqu'on explore les espoirs pour l'avenir, on constate souvent une résignation se traduisant par l'absence de projets. Quand des projets sont envisagés, ceux-ci sont presque tous irréalistes :

Une place dans la nature, j'aurais une grosse grosse maison pis le monde qui, qui pourrait pas dormir, une mauvaise journée, déprimé ou, pourrait venir là prendre un café ou parler, du monde pis, si y veulent coucher overnight y coucherait pis comme, avoir un centre là. (UC6)

Éléments propres au groupe de comparaison du milieu urbain. Les participants du groupe de comparaison en milieu urbain ont des conditions de vie beaucoup plus difficiles qui sont marquées par l'insécurité et l'instabilité.

Non, because lui boit beaucoup right $[\ldots]$. Quand qu'on était jeune $[\ldots]$ on pouvait pas aller se coucher parce que les hommes venaient après nous autres [ ... ] plusieurs fois right so toutes les fois qu'on voit de la boisson qui entre je pense holy fuck man je veux pas dormir a soir parce que j'ai peur right, je mets [ ... ] dans ma porte de chambre so qui peuvent pas rentrer. (UC 34)

Ces participants vivent dans un espace de survie, devant composer de façon quotidienne avec un logement et des services inadéquats. La dépendance aux divers services comme les soupes populaires est fréquente. Un de ces participants a tout de même réussi à améliorer sa situation en gérant sa consommation d'alcool.

Comme pour les participants des groupes expérimentaux, la présence d'enfants ou d'amis ou amies de longue date joue un rôle important :

Ah oui, on met des textes. Pis moi j'aime pas texter, je comprends pas. ... Elle me texte, là-bas de Calgary [ ... ] elle reste à Calgary. Bien, ma fille, ça fais $8-9$ ans que je ne l'ai pas vue, mais on se parle à la phone tout le temps. (UC 19)

Plusieurs participants évoquent la possibilité de retrouver leurs enfants comme une source primordiale de motivation à s'en sortir. Toutefois, tous les participants sont d'accord pour affirmer que la gestion de leur santé mentale rend toute recherche de travail impossible. 
Éléments propres au groupe de comparaison du milieu rural. Là où le milieu rural se distingue le plus est sans aucun doute par ses familles d'accueil et ses foyers de soins. Les personnes y habitant jouissent d'un sentiment de sécurité et d'appartenance sans égal en milieu urbain :

I feel safe here. (RC 17)

J'aime jaser avec le monde ici. Je suis bien ici. (RC 19)

J'aime ça ici, ils sont nice. (RC 13)

Il n'y a eu aucun déménagement de la part de ces participants pendant la durée du projet de recherche. Parmi les facteurs facilitants, les chèques d'aide sont pour plusieurs versés directement à la famille d'accueil, qui donne de l'argent de poche au participant mais qui s'assure en même temps que ce dernier aura un logement et des repas acceptables en tout temps. Le centre de santé mentale de Richibucto mène d'ailleurs son propre projet de réinsertion qui permet à ces personnes d'occuper de petits emplois. Toutefois, les mêmes difficultés de transport sont évoquées, ainsi qu'une difficulté à trouver sa place dans la communauté. Pour ceux qui ne sont pas en famille d'accueil ou en foyer de soins, on retrouve aussi le principe d'emprunts d'argent à la famille et aux amis et amies. La logique de dépendance existe même en foyer de soins.

Chez ces participants, on évoque encore des problèmes d'effets secondaires reliés à la médication. Toutefois, on retrouve chez certains d'entre eux des projets réalistes comme arrêter de fumer ou s'entrainer au gymnase.

\section{DISCUSSION}

On remarque lors de l'analyse des données une différenciation entre Moncton, milieu urbain, et la ruralité environnante telle que le prévoit la littérature. Un facteur qui explique cette différenciation des données est le phénomène de la grandeur du territoire couvert par chacun des villages, phénomène amplifié par l'absence de transports en commun, ce dont font mention Forchuk et al. (2010). Ce phénomène favorise d'abord la cohabitation des participants, par exemple lorsqu'il y a une rencontre amoureuse. Il en découle par ailleurs un manque de divertissements, ce qui cause de l'ennui, plus marquée chez les participants en milieu rural qu'en milieu urbain. L'analyse de leurs journées typiques montre qu'elles sont teintées d'ennui.

Vient également le manque de services en milieu rural et la difficulté de transport pour participer à des projets d'insertion sociale déjà identifiés (Forchuk et al., 2010; Wane et al., 2007). Fait important à signaler, les participants du groupe de comparaison étaient pour la plupart en foyer de soins, ce qui va dans le sens de la différence du bassin de logements relevé par Grigg et al. (2004). Cela explique toutefois aussi la présence de projets d'insertion sociale ainsi que les sentiments de sécurité et d'appartenance au niveau du groupe de comparaison, choses qui sont complètement absentes à Moncton où les personnes du groupe de comparaison sont livrées à elles-mêmes et habitent généralement des taudis. Non seulement ces logements en milieu urbain sont souvent insalubres mais ils sont aussi situés dans des quartiers reconnus pour la pauvreté de leurs habitants et habitantes, ce qui entraine une autre forme de stigmatisation qui vient s'ajouter à celle que les participants vivent déjà par rapport à la santé mentale. Toutefois, la logique de dépendance est très présente par rapport au foyer de soins. 
On remarque également que plusieurs participants en milieu rural pratiquent différentes formes d'art. La petite taille de l'échantillon et les données ne permettent pas d'expliquer ce phénomène mais nous avons deux hypothèses. Cette pratique artistique pourrait être liée à l'isolement et à l'ennui typique au milieu rural, mais elle pourrait aussi être liée à la prédominance de la culture acadienne. Nous avons en effet remarqué que les Acadiens et Acadiennes en milieu urbain ont eux aussi cette tendance à pratiquer une forme d'art telle qu'un instrument de musique. Cela n'est peut-être pas non plus étranger à la vocation touristique de la plupart de ces endroits, vocation qui peut amener plus de gens à vouloir partager et mettre en valeur leur culture et leur patrimoine. D'ailleurs, la plupart des participants pratiquant une forme d'art ou une autre l'ont d'abord appris de l'un de leurs parents. Toujours est-il que nous voyons dans ces diverses pratiques artistiques une opportunité particulière qui pourrait pallier aux difficultés propres au milieu rural quant à la mise en place de services proactifs et à la difficulté de lier les participants aux services (Forchuk et al., 2010).

Le sentiment d'appartenance des participants du groupe expérimental semble être moins prononcé en milieu rural qu'en milieu urbain. Rappelons-nous que ce sentiment d'appartenance était lié au choix du quartier et au fait de quitter un quartier stigmatisé pour sa pauvreté. D'une part, cette réalité n'existe pas en milieu rural, si ce n'est par une certaine rivalité entre les diverses communautés voisines. Par ailleurs, il est possible que chaque milieu rural possède déjà son identité propre, la famille habitant souvent le lieu depuis plus d'une génération. Ce sont là des paramètres qui pourraient venir enrichir les pistes d'adaptation d'un modèle de suivi intensif multidisciplinaire déjà proposées par Meyer et Morrissey (2007).

On a aussi remarqué que l'expulsion de participants est aussi un problème en milieu rural. Encore une fois, nous formulons quelques hypothèses à ce sujet. Peut-être que les écarts de conduite tels que la prostitution ou la consommation sont plus apparents au sein de petites communautés. Peut-être aussi est-ce dû à la difficulté pour l'équipe de soins de couvrir un grand territoire (Wane et al., 2007). Ces constatations, jointes aux commentaires des participants relativement à l'instabilité provoquée par un trop grand nombre d'intervenantes et d'intervenants différents pourrait suggérer une piste de solution adaptée au milieu rural qui délaisserait le modèle urbain pour adopter un modèle calqué sur les communautés rurales elles-mêmes, soit de petits noyaux d'intervenants et d'intervenantes ou des intervenantes ou intervenants seuls dédiés à chaque localité. Dans un tel cas, ce seraient les intervenantes et intervenants eux-mêmes qui bénéficieraient d'une formation multidisciplinaire et qui travailleraient en concertation les uns avec les autres.

Finalement, ce que nous retenons de cette comparaison c'est qu'au-delà des différences, on a pu observer une amélioration marquée chez les participants des deux groupes expérimentaux, tant en milieu rural qu'en milieu urbain. Cette amélioration s'est manifestée notamment au niveau de la gestion de la maladie, de la gestion de la consommation et du développement de leur autonomie. Dans les groupes de comparaison, on demeure aux prises avec la même logique de dépendance et le même phénomène de portes tournantes de l'hospitalisation au système carcéral. Tant en milieu rural qu'urbain, la longueur des listes d'attente témoigne du manque de services.

Néanmoins, le projet rural a su démontrer l'importance et la pertinence de la mise sur pied de projets d'insertion sociale et de la disponibilité de travail adapté à leur condition de santé mentale. Les symptômes de la maladie mentale et le stress lié à des échecs vécus précédemment ayant parfois mené jusqu'à l'épuisement ou à la dépression, de même que les possibilités limitées de travail qui leur laissent peu de choix quant au type d'emploi, impliquent presque assurément des conditions trop difficiles pour eux (Corbière et Durand, 
2011). Ce type de projet répond en effet à un besoin généralisé et constitue une base incontournable de l'autonomisation et de la reprise du pouvoir des participants. Le projet urbain, pour sa part, a su mettre en évidence l'intérêt d'un centre de services centralisé et accessible pour favoriser la prise en charge des participants par eux-mêmes et créer un espace de socialisation. La question de comment pourrait être implanté l'accessibilité d'un tel centre pour une population rurale demeure. Nous tenons à souligner en terminant l'importance du respect de la part des psychiatres et des intervenants et intervenantes qui a été évoqué à maintes reprises et qui a constitué, à un moment ou à un autre, soit un frein, soit un point tournant dans la vie de chacun des participants.

\section{Limites de l'étude}

La sous-étude en milieu rurale a été élaborée de façon à suivre le modèle de l'étude Chez Soi, lequel a été pensé pour un milieu urbain. Cela a permis de mettre en évidence certaines différences et certains aspects spécifiques au milieu rural, mais il n'en demeure pas moins qu'un protocole de recherche élaboré spécifiquement pour le milieu rural aurait pu donner des résultats plus précis ou plus opérationnels. Par ailleurs, il existe dans le milieu rural des clivages beaucoup plus marqués que dans le milieu urbain entre les populations francophones et anglophones. Compte tenu de la spécificité du Nouveau-Brunswick, à savoir une province où le français est reconnu comme langue officielle tout en constituant une culture minoritaire, il pourrait être pertinent de considérer de façons différentes les situations au niveau de ces deux populations. Notons que la taille de l'échantillon ne permet pas de généraliser les résultats. De même, une cueillette de données additionnelle à une date ultérieure aurait pu nous donner une meilleure perspective sur nos résultats.

\section{CONCLUSION}

En conclusion, il ne faudrait pas mettre de côté l'initiative fédérale de mettre sur pied le projet At Home / Chez Soi. L'implantation du modèle Logement d'abord implique obligatoirement un important financement. Toutefois, en regroupant les résultats des études qualitatives et quantitatives, nous disposons maintenant de données qui en démontrent l'efficacité, du point de vue tant du mieux-être des participants que de l'économie de sommes importantes que permet ce financement.

\section{RÉFÉRENCES}

Blanchet, A. et Gotman, A. (1992). L'enquête et ses méthodes : l'entretien. Paris, France : Nathan.

Corbière, M. et Durand, M. (2011). Du trouble mental à l'incapacité du travail. Québec, QC : Presses de l'Université du Québec.

Fetterman, D. M. (1998). Ethnography step-by-step (2e éd.). Thousand Oaks, CA : Sage.

Forchuk, C., Montgomery, P., Berman, H., Ward-Griffin, C., Csiernik, R., Gorlick, C., [ . . . Riesterer, P. (2010). Gaining ground, lousing ground: The paradoxes of rural homelessness. Canadian Journal of Nursing Research, 42(1), 138-152. Consulté à http://www.ingentaconnect.com/content/mcgill/cjnr/2010/00000042/00000002/art00013

Grigg, M., Judd, F., Ryan, L. et Komiti, A. (2004). Identifying marginal housing for people with a mental illness living in rural and regional areas. Australasian Psychiatry, 12(1), 36-41. doi : 10.1046/j.1039-8562.2003.02057.x

Gulcur, L., Stefancic, A., Shinn, M., Tsemberis, S. et Fischer, S. N. (2003). Housing, hospitalization and cost outcomes for homeless individuals with psychiatric disabilities participating in continuum of care and housing first programmes. Journal of Community \& Applied Social Psychology, 13(2), 171-186. doi : 10.1002/casp.723 
Henry, M. et Sermons, M. W. (2010). Geography of homelessness. Washington, DC : National Alliance to End Homelessness / Homelessness Research Institute. Consulté à http://b.3cdn.net/naeh/3953e7051f30801dc6_iim6 banq3.pdf

Henwood, B. F., Hsu, H., Dent, D., Winetrobe, H., Carranza, A. et Wenzel, S. (2013). Transitioning from homelessness: A "fresh-start" event. Journal of the Society for Social Work and Research, 4(1), 47-57. doi : 10.5243/jsswr.2013.4

Judd, K. F., Jackson, J. H., Komiti, A., Murray, G., Hodgins, G. et Fraser, C. (2002). High prevalence disorders in urban and rural communities. Australian and New Zealand Journal of Psychiatry, 36, 104-113. doi : 10.1046/j.1440-1614.2002.00986.x

Lalonde, P., Grunberg, F. et Aubut, J. (dir.). (2001). Psychiatrie clinique (Tome II). Boucherville, QC : Gaëtan Morin. Macnaughton, E. L., Goering, P. N. et Nelson, G. B. (2012). Exploring the value of mixed methods within the At Home / Chez Soi Housing First project: A strategy to evaluate the implementation of a complex population health intervention for people with mental illness who have been homeless. Canadian Journal of Public Health, 103(7), 57-62. Consulté à http://journal.cpha.ca/index.php/cjph/article/view/2962/2639

Macnaughton, E. L., Nelson, G. B., Caplan, R., Macleod, T., Townley, G., Piat, M., [ . . . ] Goering, P. N. (2013). Evaluation of the Mental Health Commission of Canada's At Home / Chez Soi project: Cross-site report on consumer narratives at baseline and 18-month follow-up. Ottawa, ON : Mental Health Commission of Canada, National Qualitative Research Team for the At Home / Chez Soi Project.

Marx, A. J., Test, M. A. et Stein, L. I. (1973). Extrohospital management of severe mental illness: Feasibility and effects of social functioning. Archives of General Psychiatry, 29, 505-511. doi : 10.1001/archpsyc.1973.04200040051009

Meyer, P. S. et Morrissey, J. P. (2007). A comparison of Assertive Community Treatment and Intensive Case Management for patients in rural areas. Psychiatric Services, 58(1), 121-127. Consulté à http://journals.psychiatryonline.org/ data/Journals/PSS/3790/07ps121.pdf

New Brunswick Department of Post-Secondary Education, Training and Labour. (2012). New Brunswick regional profiles: Southeast profile. Consulté à http://www2.gnb.ca/content/dam/gnb/Departments/petl-epft/PDF/Publications/ SoutheastProfile.pdf

O’Connell, M. J., Kasprow, W. et Rosenheck, R. (2009). Direct placement versus multistage models of supported housing in a population of veterans who are homeless. Psychological Services, 6(3), 190-201. doi : 10.1037/a0014921

Padgett, D. K., Stanhope, V., Henwood, B. F. et Stefancic, A. (2011). Substance use outcomes among homeless clients with serious mental illness: Comparing Housing First with treatment first programs. Community Mental Health Journal, 47(2), 227-232. doi : 10.1007/s10597-009-9283-7

Paillé, P. et Mucchielli, A. (2003). L'analyse qualitative en sciences humaines et sociales. Montréal, QC : Armand Colin.

Polvere, L., Macnaughton, E. et Piat, M. (2013). Participant perspectives on Housing First and recovery: Early findings from the At Home / Chez Soi project. Psychiatric Rehabilitation Journal, 36(2), 110-112. doi : 10.1037/h0094979.

Prabhu, R. et Browne O. M. (2007). A recovery-based outreach program in rural Victoria. Australasian Psychiatry, 15(2), 120-127. doi : 10.1080/10398560701196729

Prévost, N. et Nolin, D. (2011). Rapport sur les récits de vie de base. Moncton, NB : Projet At Home / Chez Soi. Consulté à http://www.umoncton.ca/crde/files/crde/wf/wf/pdf/Rural et Moncton Rapport final sur le recit de vie de base - copie.pdf

Rosenheck, R., Kasprow, W., Frisman, L. et LiuMares, W. (2003). Cost-effectiveness of supported housing for homeless persons with mental illness. Archives of General Psychiatry, 60(9), 940-951. doi :10.1001/archpsyc.60.9.940.

Société américaine de psychiatrie. (2002). Manuel diagnostique et statistique des troubles mentaux. Paris, France : Elsevier Masson.

Stefancic, A. et Tsemberis, S. (2007). Housing First for long-term shelter dwellers with psychiatric disabilities in a suburban county: A four-year study of housing access and retention. Journal of Primary Prevention, 28(3-4), 265-279. doi : 10.1007/s10935-007-0093-9

Tsemberis, S. (1999). From streets to homes: An innovative approach to supported housing for homeless adults with psychiatric disabilities. Journal of Community Psychology, 27, 225-241.

Wane, J., Owen, A., Sood, L., Bradley, S. et Jones, C. (2007). The effectiveness of rural assertive outreach: A prospective cohort study in an English region. Journal of Mental Health, 16(4): 471-482. doi : 10.1080/09638230701483129 